\title{
Importancia del cepillado antes de acostarse en la prevención de la caries. Estudio en una población infantil
}

\author{
Ruiz Sedano TE*, Vicente Barrero $M * *$, Limiñana Cañal $J M^{* * *}$, Knezevic $M^{* * * * *}$, \\ Henández Perdomo O*****, Ruiz Sedano $M^{* * * * * * *, ~ S e r r a ~ M a j e m ~ L * * * * * * * * ~}$
}

\section{RESUMEN}

En el presente trabajo nos planteamos si sería imprescindible el cepillado tres veces al día y, en caso contrario, en qué momento del día sería más importante la eliminación mecánica de la placa. Para ello, se planteó un estudio transversal correspondiente a 214 escolares de 6-12 años en el periodo comprendido en el curso 2004-5. A cada niño se le practicó el "Examen parcial o tipo III" recomendado por la Federación Dental Internacional, como procedimiento estándar para un estudio de prevalencia de caries.

Hemos obtenido los siguientes resultados:

1. El cepillado de dientes antes de acostarse es un factor protector de la presencia de caries, siendo el correspondiente odd-ratio ajustado de 0.339 (intervalo de confianza al 95\% [0.159; 0.722]).

2. El consumir azúcar más de una vez una vez al día tiene más riesgo de aparición de caries que si consumen una vez al día, siendo el correspondiente odd-ratio ajustado de 1.998 (intervalo de confianza al 95\% [1.090; 3.665]). 3. Los niños que acuden a un colegio público tienen más riesgo de presentar caries que los que asisten a un colegio privado. Siendo el correspondiente odd-ratio ajustado de 2.019 (intervalo de confianza al 95\% [1.105; 3.691]).

4. Se cumplen en la población estudiada los objetivos marcados sobre caries dental por la OMS para el año 2000.

Palabras clave: Caries, Cepillado.

\section{SUMMARY}

In the present study we are questioning if the three time a day tooth brushing is really essential and if it is the opposite case, what time of the day would be most important for mechanical elimination of dental plaque. For this purpose we have planed a transversal study on 214 pupils aged 6 to 12 years in the period of 2004-05 scheduled school coarse. On each of them was applied the "Partial Exam type III" which is recommended from International Dental Federation and which is considered as standard procedure on prevalence of dental caries study.

We obtained following results:

1. Tooth brushing by night before sleeping is a favourable protection factor from dental caries, being correspondent odd-ratio adjusted on 0.339 (interval of confidence is 95\% [0.159; 0.722]).

2. Consuming the sugar more than one time a day causes higher risk for dental caries apparition than one time a day consumption of sugar, being correspondent odd-ratio adjusted on 1.998 (interval of confidence is 95\% [1.090;3.665]).

3. The children attending public schools have higher risk of dental caries presence than the children attending private schools being correspondent odd-ratio adjusted on 2.019 (interval of confidence is 95\% [1.105;3.691]). 4. The objectives on dental caries marked from WHO are carried out on the studied population.

Key words: Dental caries. Tooth brushing.

Fecha de recepción: Junio 2006.

Aceptado para publicación: Octubre 2006. 


\begin{tabular}{|c|c|}
\hline * & Licenciada en Farmacia. Máster en Nutrición y Seguridad Alimentaria \\
\hline ** & $\begin{array}{l}\text { Servicio de Estomatología, Cirugía Oral y Máxilo-facial. Profesor Asociado de la Universidad de Las } \\
\text { Palmas de Gran Canaria. }\end{array}$ \\
\hline *** & Catedrático de Estadística de la Universidad de Las Palmas de Gran Canaria. \\
\hline$* * * *$ & Servicio de Estomatología, Cirugía Oral y Máxilofacial. \\
\hline ***** & Odontoestomatólogo de Área de Gran Canaria. \\
\hline$* * * * * *$ & Odontóloga. \\
\hline$* * * * * * *$ & $\begin{array}{l}\text { Catedrático de Medicina Preventiva y Salud Pública. Universidad de Las Palmas de Gran Canaria } \\
\text { Presidente de la Sociedad Española de Nutrición Comunitaria. }\end{array}$ \\
\hline \multicolumn{2}{|r|}{$\begin{array}{l}\text { Ruiz Sedano TE, Vicente Barrero M, Limiñana Cañal JM, Knezevic M, Hernández Perdomo O, Ruiz Sedano M, } \\
\text { Serra Majem L. Importancia del cepillado antes de acostarse en la prevención de la caries. Estudio en una } \\
\text { población infantil. Av. Odontoestomatol 2007; } 23 \text { (1): 45-50. }\end{array}$} \\
\hline
\end{tabular}

\section{INTRODUCCIÓN}

La caries es el proceso patológico más frecuente del diente, condicionado por numerosos factores. Keyes en la década de los 60 estableció el carácter infeccioso y transmisible de la caries, en trabajos experimentales (1). Además estableció el carácter multifactorial de la caries, haciendo responsable de su aparición a la confluencia de la microflora, el substrato (dieta) y el huésped-diente. Posteriormente se han añadido otros factores secundarios, pero imprescindibles en la etiopatogenia de la caries (2): tiempo de actuación para que intervengan los otros factores (3); la saliva y los factores que dependen de ella, como la capacidad buffer, el $\mathrm{pH}$, las inmunoglobulinas (4); la edad del individuo, fundamental en la homeostasia y la inmunidad (5); así como los factores de ingeniería biodental (6). Como vemos, aún hoy día se sigue discutiendo sobre diferentes aspectos etiopatogénicos de la caries, pero el papel etiológico primordial de la placa bacteriana es incuestionable. Partiendo de esta premisa, en el presente trabajo nos planteamos si sería imprescindible el cepillado tres veces al día y, en caso contrario, en qué momento del día sería más importante la eliminación mecánica de la placa.

\section{MATERIAL Y MÉTODOS}

\section{Muestra}

Durante el curso académico 2004-5, estudiamos un total de 214 escolares de edades comprendidas en- tre los 6 y los 12 años, que acudieron, previa cita, al Centro de Salud de Canalejas en Las Palmas de Gran Canaria. La exploración fue realizada por el mismo odontoestomatólogo de zona y la higienista dental, pertenecientes ambos al sistema público de salud, anotándose los datos en la historia clínica dental.

\section{Criterios de inclusión y exclusión}

Se incluyeron todos los escolares que de forma aleatoria y consecutiva iban siendo citados en el Centro de Salud, previo consentimiento informado por parte del adulto que acompañaba al niño a dicha cita. Fueron excluidos aquellos niños que en el momento de la exploración eran portadores de brackets de y/o aparatos removibles de ortodoncia, ya que suponíamos que podían sesgar los resultados.

\section{Diseño metodológico}

Se trata de un estudio transversal correspondiente a los 6-12 años en el periodo comprendido en el curso 2004-5.

\section{Examen clínico-odontológico}

Los escolares fueron explorados en el gabinete odontoestomatológico del Centro de Salud. Se abrió una historia médico-odontológica en la que se consideraron las siguientes variables: Edad, sexo, tipo de colegio (público o privado), hábitos de higiene bu- 
codental (número y momento de los cepillados diarios, cuidado de las encías sangrantes, uso de enjuague bucal y dentífricos fluorados, tipo de cepillo y pasta dentífrica), hábitos nocivos (respiración bucal, chuparse el dedo, morderse las uñas, ausencia de cepillado de dientes inmediatamente posterior a la ingesta de azúcar), ingesta de hidratos de carbono refinados, bien en forma de productos de bollería para completar su dieta, bien en forma de dulces y caramelos (azúcares retentivos) o en refrescos azucarados (azúcares no retentivos), frecuencia de asistencia a la consulta dental, existencia de caries (número de caries, número de superficies afectadas, localización), existencia de gingivitis y otros factores (tratamientos fluorados, maloclusión, tipo de agua de bebida).

La toma de datos se realizó mediante exploraciones bucales practicadas por el mismo examinador, en equipo dental, con buena iluminación, espejo plano y sonda dentales. A cada niño se le practicó el "Examen parcial o tipo III" recomendado por la Federación Dental Internacional (7), como procedimiento estándar para un estudio de prevalencia de caries. Este comprende el uso de espejo y sonda, así como la mejor iluminación que se pueda disponer. Se siguieron los criterios tradicionales de diagnóstico de caries (8).

Los índices de caries estudiados se han elegido en función de la finalidad de la encuesta, sin ignorar que son muchos los índices aplicables, hemos utilizado los, que según nuestra opinión, eran los mas apropiados para la misma (9).

\section{Análisis estadísticos}

Las variables categóricas se resumieron en porcentajes y las variables numéricas, al no darse los supuestos de normalidad, se resumieron como medianas y percentiles 5 y 95. Para ver si existe asociación entre presencia o no de caries y las variables categóricas se aplicará el test de independencia de la chicuadrado. En orden a investigar los factores asociados con la presencia o no de caries se obtuvieron las odds-ratios crudas para aquellos que mostraron diferencias estadísticamente significativas los cuales se estimaron mediante intervalos de confianza al 95\%. Todos los factores que individualmente se asociaron con la presencia de caries fueron incluidos en un análisis de regresión logística y mediante un proceso de selección de variables basado en el test de la razón de verosimilitudes, se determinaron los factores que se asocian de forma independiente con la presencia de caries. Del modelo de regresión logística obtenido se dedujeron las correspondientes oddsratio ajustadas. También se utilizaron las pruebas no paramétricas de Mann-Whitney para dos muestras independientes y de Kruskal-Wallis para más de dos muestras independientes.

Un contraste de hipótesis se considera significativo cuando el correspondiente p-valor sea inferior a 0,05. Los datos se analizaron con el paquete estadístico SPSS (versión 13.0 para Windows).

\section{RESULTADOS}

De los 214 niños de la muestra, $111(51,87 \%)$ eran niños y 103 (48,13\%) niñas. Del total, 133 (62,15\%) recibían enseñanzas en colegios públicos y 81 $(37,85 \%)$ en colegios privados. Al observar la existencia o no de caries $93(43,46 \%)$ tenían caries mientras que $133(62,15 \%)$ no tenían.

El CAOd, en el total del grupo es 1,63. Si consideramos las superficies afectadas CAOs, tenemos 1,88. A los 6 años, la media del CAOd es 1,7 y el CAOs 2,6. A los 12 años, encontramos un CAOd de 1,24 y un CAOs de 1,34.

Para elegir cuáles son las posibles variables que pueden estar asociadas con la presencia o no de caries en nuestro fichero de datos, realizamos un análisis crudo mediante pruebas de independencia de la chicuadrado, tomando como medida de asociación (para aquellas variables que resultaron estadísticamente significativas) las odd-ratios crudas las cuales se estimaron mediante intervalos de confianza al 95\%. Los resultados obtenidos se resumen en la Tabla 1, donde podemos comprobar existe asociación entre las variables cepillado antes de acostarse, tipo de colegio y el consumo de azúcar con la presencia de caries, mientras que no existe con el resto de las variables. Esto lo podemos comprobar con la inspección de los intervalos de confianza de los odds-ratios, viendo si el valor 1 pertenece o no al intervalo o bien observando si los valores de la $\chi^{2}$ son significativos. 
AVANCES EN ODONTOESTOMATOLOGÍA

Vol. 23 - Núm. 1 - 2007

\section{TABLA 1.- ESTUDIO DE ASOCIACIÓN ENTRE VARIABLES CATEGÓRICAS Y LA PRESENCIA O NO DE CARIES}

\begin{tabular}{|l}
\hline Factor \\
FRECUENCIA DE CEPILLADO
\end{tabular}

Caries sí (\%)

$\mathrm{P} *$

$\mathrm{OR}^{* *}$

IC***

- Nunca

- 1 al día

14

51

- 2 al día

97

- 3 al día

52

CePillado antes de acostarse

- No

- Sí

TIPO PASTA

- Sin flúor

- Con flúor

ENFERMEDADES BUICODENTALES

- Mala oclusión

- Sana

TIPO DE AGUA

- Con gas

- Sin gas

- Otras

ASISTENCIA AL DENTISTA

- Nunca

- Anualmente

86

128

SEXO

- Niño

- Niña

CONSUMO DE AZÚCAR

- >1 vez al día

- 1 vez al día

76

111

103

24

15

$27(67,5)$

$66(37,9)$

$8(57,1)$

0,166

$24(47,1)$

$45(46,4)$

$16(30,8)$

40

174

82

132

$40(48,8)$

$53(40,2)$

0,001

3,40

$(1,64-7,05)$

50

149

$26(52,0)$

$59(39,6)$

0,086

0,216

0,216

$75(43,9)$

$11(45,8)$

$5(33,3)$

0,708

$35(40,7)$

$58(45,3)$

0,504

$44(39,6)$

$49(47,6)$

0,144

$68(49,3)$

$25(32,9)$

0,02

1,98

(1,11-3,55)

TiPo DE COLEGIO

- Público

133

- Privado

0,004

2,34

$(1,31-4,18)$

* Determinado por el test de independencia de la $\chi^{2}$

** Odd-ratio estimado.

*** Intervalo de confianza para la odd-ratio al 95\% 
1. El cepillado de dientes antes de acostarse es un factor protector de la presencia de caries, siendo el correspondiente odd-ratio ajustado de 0.339 (intervalo de confianza al 95\% [0.159; 0.722]).

2. El consumir azúcar más de una vez una vez al día tiene más riesgo de aparición de caries que si consumen una vez al día, siendo el correspondiente odd-ratio ajustado de 1.998 (intervalo de confianza al 95\% [1.090; 3.665]).

3. Los niños que acuden a un colegio público tienen más riesgo de presentar caries que los que asisten a un colegio privado. Siendo el correspondiente odd-ratio ajustado de 2.019 (intervalo de confianza al 95\% [1.105; 3.691]).

\section{DISCUSIÓN}

Los objetivos de la OMS en su programa de salud para todos en el año 2000, tiene como misión establecer una prevalencia de caries del $50 \%$ a los 6 años y un índice CAOd menor de 3 a los 12 años (10).

Tomando toda la población infantil estudiada, hemos obtenido una prevalencia del $43,46 \%$ y un CAOd de 1,63. Si nos centramos en los 6 años, la prevalencia es del $50 \%$ y el CAOd 1,7 . A los 12 años la prevalencia es del $47,1 \%$ y el CAOd 1,24 . Por tanto, estos índices cumplen los objetivos marcados por la OMS.

El 93,45\% de los escolares estudiados se cepillan los dientes al menos una vez al día, en contraste con estudios realizados a nivel nacional hace 15-20 años, que revelaban una cifra baja de higiene dental al día, entre el 1 y el 50\% (11-15) (Tabla 2). En estos últimos años es cuando se han iniciado los programas preventivos en salud bucodental, por lo parece evidente la relación causa-efecto con respecto a la mejora en las condiciones sanitarias.

Al comparar el número de cepillados al día con el CAOd y el CAOs, no obtuvimos datos significativos con uno o dos cepillados al día, pero sí encontramos un hallazgo significativo en el CAOd $(p=0,04)$ y CAOs $(p=0,041)$, cuando se comparaban aquellos niños que no se cepillaban nunca los dientes con aquellos que lo hacían tres veces al día.

Encontramos también un hallazgo interesante en cuanto al momento del cepillado, ya que en la asociación de variables era muy significativo el cepillado antes de acostarse con respecto a la prevención de caries. En el estudio de Caldes y cols de 2005 (16) en niños de 12 años, no se encontró relación entre el número de cepillados al día, aunque en dicho estudio tampoco se valoró individualmente el valor del cepillado antes de acostarse.

\section{CONCLUSIONES}

A la luz de los resultados obtenidos hemos podido concluir los siguientes puntos:

1. Se cumplen en la población estudiada los objetivos marcados sobre caries dental por la OMS para el año 2000.

TABLA 2.- NIVEL DE HIGIENE DENTAL, CONSIDERANDO AL MENOS UN CEPILLADO AL DÍA

\begin{tabular}{|lcccc|}
\hline Autor & Año & Población & Edad & Higiene dental \\
\hline Baca & 1989 & Jaén & General & $1,00 \%$ \\
Barranquero & 1980 & País Vasco & $11-12$ años & $55,00 \%$ \\
Casas & 1989 & Huesca & 12 años & $43,83 \%$ \\
Rodríguez Baciero & 1980 & Bilbao & Niños & $41,35 \%$ \\
Rodríguez Contreras & 1987 & Granada & $11-13$ años & $37,46 \%$ \\
Villalba & 1987 & Alicante & $7-14$ años & $26,63 \%$ \\
Presente estudio & 2005 & Las Palmas GC & $6-12$ años & $93,45 \%$ \\
\hline
\end{tabular}


2. Al contrario de lo esperado, no existe asociación entre el número de cepillados y la existencia de enfermedades bucodentales. En cambio sí es estadísticamente significativo el cepillado antes de acostarse, tanto en el número de dientes, como en el número de superficies afectadas.

3. Al comparar el número de cepillados al día con el CAOd y el CAOs, no se obtenían datos significativos con uno o dos cepillados al día, pero sí encontramos un hallazgo significativo en el CAOd $(p=0,04)$ y CAOs $(p=0,041)$, cuando se comparaban aquellos niños que no se cepillaban nunca los dientes con aquellos que lo hacían tres veces al día.

\section{BIBLIOGRAFÍA}

1. Keyes PH. The infectious and transmissible nature of experimental dental caries. Arch Oral Biol, 1960; 1: 304-20.

2. Llamas Cadaval R, Jiménez Planas A, Sánchez Rosario M, Sánchez Barriga R. Los seis factores actuales en la etiopatogenia de la caries. Rev Eur Odontoestomatol 1994; 6: 221-4.

3. Newbrun E. Cariología. $1^{a}$ ed. Mexico, 1984: 2976.

4. Nikiforuk G. Understanding dental caries. Nueva York, Karges Press 1985; I: 72-81.

5. Miles AE. Cambios por la edad en los tejidos dentarios. En: Cohen B y Kramer IR. Fundamentos científicos de odontología. Barcelona. Saalvat Editores 1981: 435-49.

6. Grippo JO, Masi JV. The role of stress corrosion and piezoelectricity in the formation of root caries. En: Foster KR. Biogeng Conf, 12-13 marzo 1987. University of Pennsylvania, Philadelphia, 93-5.

7. FDI. Informe técnico n³. 1975.

8. Radike AW. Criteria for diagnosis of dental caries. En: Proceedings of the Conference of the Clinical testings of Cariostatic Agents. Am Dent Assoc 1979; 98: 936-7.

9. OMS. Etiología y prevención de la caries dental. Serie de informes técnicos nº 494, Ginebra, 1972.

10. OMS. Serie de informes técnicos 713. Métodos y prevención de las enfermedades bucales 1984; 7: 53.

11. Baca P. Indices de caries en una comunidad gitana. Arch Odontoestmat 1989; 5: 527-31.

12. Barranquero M. Aplicación práctica en el área de salud oral de un programa de salud oral de un programa de educación para la salud en escolares. Rev Eur Odontoestomatot 1980; 2: 175-80.

13. Casas VM, Gállego RM. Hábitos higiénicos bucodentales en escolares de 12 años de la ciudad de Huesca. Atención Primaria 1992; 10: 821-4.

14. Rodríguez R. Prevalencia de la caries dental en una zona costera granadina. Rev Med Clin Navarra 1987; 31: 75-8.

15. Villalba JR, Rico R, Pérez-Gaeta JC, Casanova J. El problema de la caries dental en la edad escolar: un estudio transversal. Rev Hig San Pub, 1985; 59: 1215-22.

16. Caldés S, Cea N, Crespo P, Díez V, Espino A. ¿Una intervención educativa en niños de doce años de Madrid modifica sus conocimientos y hábitos de higiene bucodental? Av Odontoestomatol 2005; 21: 149-57.

\section{CORRESPONDENCIA}

Mario Vicente Barrero

Alcalde Henríquez Pitti, 13, $1^{\circ}$ izq

35400 Arucas

Telf.: 928257791

Fax: 928634736

Email: mmvicente@wanadoo.es 